\title{
Review Article \\ Determinants for Substrate Specificity of Protein Phosphatase 2A
}

\author{
Andrew M. Slupe, Ronald A. Merrill, and Stefan Strack \\ Department of Pharmacology, University of Iowa, 2-432 BSB, Iowa City, IA 52242, USA \\ Correspondence should be addressed to Stefan Strack, stefan-strack@uiowa.edu
}

Received 4 March 2011; Accepted 28 April 2011

Academic Editor: Hemant Paudel

Copyright ( $) 2011$ Andrew M. Slupe et al. This is an open access article distributed under the Creative Commons Attribution License, which permits unrestricted use, distribution, and reproduction in any medium, provided the original work is properly cited.

\begin{abstract}
Protein phosphatase 2A- (PP2A-) catalyzed dephosphorylation of target substrate proteins is widespread and critical for cellular function. PP2A is predominantly found as a heterotrimeric complex of a catalytic subunit (C), a scaffolding subunit (A), and one member of 4 families of regulatory subunits (B). Substrate specificity of the holoenzyme complex is determined by the subcellular locale the complex is confined to, selective incorporation of the B subunit, interactions with endogenous inhibitory proteins, and specific intermolecular interactions between PP2A and target substrates. Here, we discuss recent studies that have advanced our understanding of the molecular determinants for PP2A substrate specificity.
\end{abstract}

\section{Introduction}

Cellular adaptation requires biochemical processes including post-translational mechanisms to modify existing proteins. Catalyzed by opposing kinases and phosphatases, reversible phosphorylation of serine, threonine, and tyrosine residues is now appreciated as a fundamental regulatory mechanism with the majority of phosphorylation (>99\%) occurring on serine and threonine residues $[1,2]$. Due to their untapped therapeutic potential, protein phosphatases have been identified as promising targets for xenobiotic manipulation through rational drug design (reviewed in [3-6]). In particular, the ubiquitously expressed protein phosphatase 2A (PP2A) has been proposed as a target for the treatment of a number of pathologies ranging from neurodegenerative diseases such as Alzheimer disease to a variety of neoplasias [7-9].

Compared to other members of the phosphoprotein phosphatase (PPP) superfamily of serine/threonine phosphatases, a detailed understanding of the mechanism by which PP2A recognizes substrates and mediates site-specific dephosphorylation remains to be developed. Sequence and structural homology of the catalytic subunits of PPP family members has revealed a conserved catalytic mechanism in which a divalent metal cation activates a water molecule to hydrolyze phospho-serine/threonine without the formation of a phosphoenzyme intermediate (reviewed in [10-12]).
Despite a shared catalytic mechanism, substrate specificity within the PPP family is mediated by distinct mechanisms of substrate recognition. For example, the PPP calcineurin (also known as protein phosphatase 2B) has been shown to interact with two consensus sequences, PxIxIT and LxVP, found on nonsubstrate-interacting proteins and target substrates (reviewed in $[11,13]$ ). For protein phosphatase-1 (PP1), substrate specificity is conferred by incorporation of PP1-interacting proteins via a conserved docking motif with a general consensus sequence of $\mathrm{RVxF}$ (reviewed in $[11,12]$ ). At present, consensus sequences in PP2A substrates have not been identified. This review will focus on our emerging understanding of PP2A substrate specificity, which appears to involve additive effects of multiple discrete interactions.

PP2A is a highly conserved serine/threonine phosphatase which, depending on the tissue of origin and cell type, may account for up to $1 \%$ of cellular protein and the majority of serine/threonine phosphatase activity [14]. The physiological functions of PP2A have been implicated in all facets of cellular existence (reviewed in [15]). Further, PP2A functions as a critical tumor suppressor whose interruption leads to proliferative diseases [14]. The heterotrimeric holoenzyme is composed of a catalytic subunit (C) a scaffold subunit (A) and one member of four families of regulatory subunits (B) (Figure 1). The diversity of PP2A heterotrimers is achieved through expression of two $\mathrm{C}$ subunits, two A subunits and approximately fifteen B subunits in vertebrates. The B 
subunits are derived from four diverse gene families $\left(B, B^{\prime}\right.$, $\mathrm{B}^{\prime \prime}$, and $\mathrm{B}^{\prime \prime \prime}$ ) that have little sequence similarity between families but maintain high sequence similarity within families. The B family (B55, PR55, PPP2R2) of regulatory subunits consists of four genes $(\alpha, \beta, \gamma, \delta)$, the $\mathrm{B}^{\prime}$-family (B56, PR61, PPP2R5) is comprised of five isoforms $(\alpha$, $\beta, \gamma, \delta, \varepsilon)$, the $\mathrm{B}^{\prime \prime}$ family (PR72, PPP2R3) includes three isoforms $(\alpha / \mathrm{PR} 72 / 130, \beta / \mathrm{PR} 59, \gamma / \mathrm{PR} 48)$, and the $\mathrm{B}^{\prime \prime \prime}$ family (PR93/PR110) is made up of three proteins (SG2NA, striatin, and $\mathrm{mMOB} 1)$. There is some controversy as to whether the $\mathrm{B}^{\prime \prime \prime}$ family members, most notably SG2NA, are bona fide $\mathrm{PP} 2 \mathrm{~A}$ regulatory subunits that always associate with the AC dimer or whether they are merely regulated by association with the PP2A dimer. Given the large number of PP2A subunits, it is thought that each cell expresses a dozen or more distinct holoenzyme complexes which act on a diverse array of substrates. PP2A holoenzyme diversity has been the subject of several excellent papers $[14,15,18]$.

Like PP1, regulatory subunit incorporation is thought to dictate the substrate specificity of the PP2A complex, however, only recently have molecular studies begun to develop insight into the mechanism by which the regulatory subunit acts [15]. The results from recent studies suggests a multitiered mechanism wherein PP2A substrate specificity arises from (1) subcellular localization of PP2A defined by the B subunit, (2) selective holoenzyme assembly by posttranslational modification, (3) interaction with specific endogenous inhibitors, (4) interactions between the B subunit and phosphosubstrates at sites distant from the active site, and (5) B-subunit residues which infiltrate the catalytic cleft of the $\mathrm{C}$ subunit. This paper will provide a summary of these studies and how the understanding of the determinants of PP2A substrate specificity has advanced.

\section{Mechanisms of Substrate Specificity}

2.1. Subcellular Localization of the Holoenzyme Complex. The heterotrimeric holoenzyme is targeted to discrete subcellular locales dictated in part by which B-regulatory subunit is incorporated. The localization imparted by the B-regulatory subunit dictates the spatial sphere of influence of the holoenzyme complex for potential substrates. This mechanism of targeting PP2A activity is highlighted by extensive studies of the B family of regulatory subunits. For instance, the B family regulatory subunits target the holoenzyme to different cellular compartments in the brain [19]. Specifically, B $\alpha$ and $\mathrm{B} \beta$ are primarily cytosolic where as the $\mathrm{B} \gamma$-regulatory subunit associates with a detergent-resistant protein fraction consistent with an interaction at the cytoskeleton [19].

Similar diversity has been observed in the $\mathrm{B}^{\prime}$ family of regulatory subunits. A C-terminal nuclear export signal common to $\mathrm{B}^{\prime} \alpha, \mathrm{B}^{\prime} \beta$, and $\mathrm{B}^{\prime} \varepsilon$ which, when these regulatory subunits are incorporated into the PP2A holoenzyme, results in cytoplasmic localization of the heterotrimer[20]. B' $\delta$ and $\mathrm{B}^{\prime} \gamma$ isoforms lack a similar sequence and are found primarily in the nucleus [21]. In cardiomyocytes, $\mathrm{B}^{\prime} \alpha$ interacts with the protein ankyrin-B through its $\mathrm{C}$-terminus which leads to localization at the cardiac M-line [22]. $\mathrm{B}^{\prime} \gamma$ on the other hand has been shown to target the holoenzyme complex

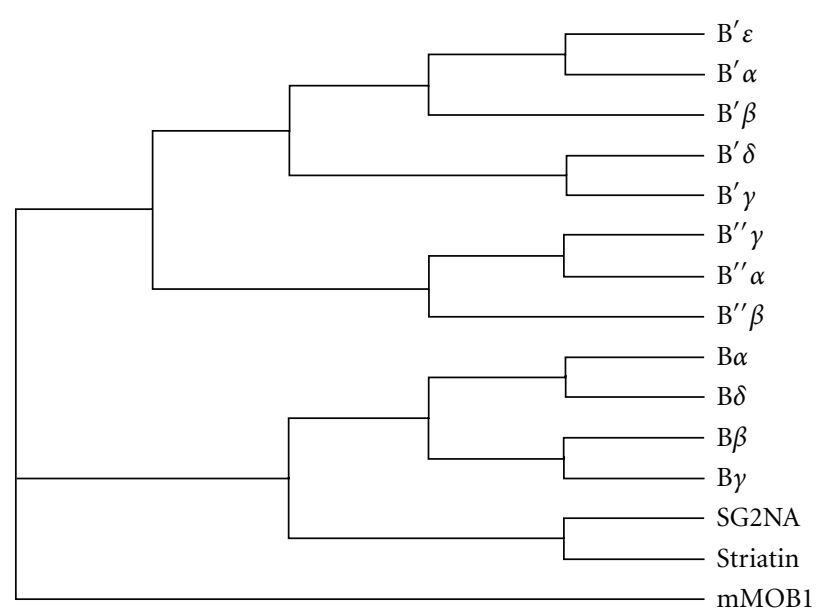

(a)

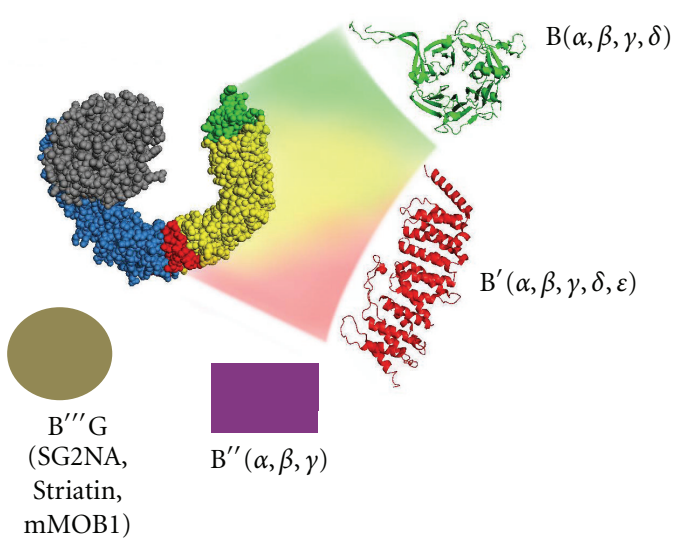

(b)

FIGURE 1: Diversity of the B-regulatory subunits and the structure of the PP2A holoenzyme complex. (a) Cladogram of the human PP2A B-regulatory subunits generated using ClustalW [16] and shown with Dendroscope [17]. (b) Structure of the dimeric A scaffold subunit and $C$ catalytic subunit (gray). The shaded portions of the A scaffold subunit indicate the known docking sites for the $B$ regulatory subunits; green (HEAT repeat 1 ) for the $B$ family of regulatory subunits, red (HEAT repeat 8) for the B' family of regulatory subunits, and yellow (HEAT repeat 2-7) for the docking site area common for the B and $\mathrm{B}^{\prime}$ families. Cartoon structure of the $\mathrm{B}$ (green ribbon), B' (red ribbon), B" (purple rectangle), and $\mathrm{B}^{\prime \prime \prime}$ (tan circle) families of regulatory subunits. Cartoon structures were generated using the Protein Data Bank accession codes 3DW8 and 2 NPP.

to subnuclear structures in cardiomyocytes where PP2A/B' $\gamma$ may regulate gene expression [23]. Loss of proper subcellular targeting of PP2A has been implicated in the biogenesis and aggressive phenotype of neoplastic growths. Specifically, a truncated form of $\mathrm{B}^{\prime} \gamma\left(\Delta \mathrm{B}^{\prime} \gamma\right)$, has been isolated from a melanoma cell line wherein the $\mathrm{PP} 2 \mathrm{~A} / \Delta \mathrm{B}^{\prime} \gamma$ complex is targeted to the trans-golgi network, blunts p53 responsiveness, contributes to genetic instability and increases metastatic motility [24-27].

Further complexity arises from differing localization imparted by alternative splicing of regulatory subunit genes. 
This was first observed in a member of the $\mathrm{B}$ family, $\mathrm{B} \beta$. Two neuron-specific isoforms of $\mathrm{B} \beta, \mathrm{B} \beta 1$, and $\mathrm{B} \beta 2$, are generated by the use of an alternative $5^{\prime}$ exon which results in the production of a divergent $\mathrm{N}$-terminal extension on $\mathrm{B} \beta 2[28,29]$. The $\mathrm{N}$-terminal extension of $\mathrm{B} \beta 2$ directs the holoenzyme complex to the outer mitochondrial membrane (OMM) by targeting the mitochondrial translocase complex and forming an abortive complex resistant to import into the mitochondrial matrix $[28,30]$. The OMMdirected $\mathrm{PP} 2 \mathrm{~A} / \mathrm{B} \beta 2$ complex promotes fragmentation of the mitochondria reticulum and increases cell susceptibility to proapoptotic insults through an unknown mechanism [31]. Alternative splicing of B-regulatory subunits directing subcellular localization of the PP2A holoenzyme has also been observed in both the $\mathrm{B}^{\prime}$ and $\mathrm{B}^{\prime \prime}$ families. Two $\mathrm{B}^{\prime} \varepsilon$ isoforms differ by the inclusion or exclusion an $\mathrm{N}$-terminal nuclear localization signal leading to isoform-specific nuclear or cytoplasmic localization [32]. The murine-specific B" family member, $\mathrm{B}^{\prime \prime} \delta$, is also subject to alternative splicing leading to isoform-specific nuclear or cytoplasmic localization through an as yet unidentified mechanism [33].

Localization is also imparted by interactions between the PP2A holoenzyme complex and other effectors. For example, the interaction between $\mathrm{PP} 2 \mathrm{~A} / \mathrm{B}^{\prime}$ and shugoshin during meiosis is crucial for spatial and temporal regulation of sister chromatid disjunction. During meiosis, the cohesin complexes, which link the arms of bivalent chromosomes and the centromeres of sister chromatids, must be released in a stepwise fashion by the protease separase; the cohesin complex is firstly hydrolyzed along the arms of the bivalent chromosomes for completion of anaphase I and secondly at the centromeres of sister chromatids for completion of anaphase II. During anaphase I, the centromeric cohesin complex is protected from separase-dependent proteolysis by shugoshin $[34,35]$. Shugoshin recruits $\mathrm{PP} 2 \mathrm{~A} / \mathrm{B}^{\prime}$ to the centromere which likely results in dephosphorylation of the cohesin complex leading to protection of the cohesin complex from separase-dependent proteolysis [36, 37]. Through cocrystallization, $\mathrm{Xu}$ and colleagues, have revealed that dimeric human shugoshin 1 interacts with $\mathrm{PP} 2 \mathrm{~A} / \mathrm{B}^{\prime} \gamma$ through a coiled-coil region across a broad composite surface of the $C$ and $B^{\prime} \gamma$ subunits [38]. Other PP2A-effector interactions have recently been reviewed elsewhere [14].

\subsection{Selective Holoenzyme Assembly and Activation. Incor-} poration of specific regulatory subunits is influenced by reversible posttranslational modification of the $\mathrm{C}$ subunit. Many groups have shown that the $\mathrm{C}$-terminus of the $\mathrm{C}$ subunit is modified through phosphorylation and methylation on Y307 and L309, respectively [39-46]. Phosphorylation of Y307 is catalyzed by src kinase and is likely opposed by PP2A-catalyzed autodephosphorylation of this phosphotyrosine [46]. Phosphorylation of Y307 selectively inhibits recruitment of the B family and some $\mathrm{B}^{\prime}$ family members to the dimeric $\mathrm{AC}$ complex whereas $\mathrm{B}^{\prime \prime}$ recruitment is not effected [44].

Methylation of the C subunit at the C-terminal L309 is catalyzed by the protein phosphatase methyltransferase (PPM1) and is opposed by the phosphatase methylesterase
(PME-1) [47-51]. Reversible methylation of PP2A is absolutely critical as knocking-out PME-1 in mice changes the phosphoproteome and results in early perinatal lethality [52]. Further, methylation of the $\mathrm{C}$ subunit is a dynamic process which plays a role in cellular response to acute stimuli [53]. The recruitment of the B subunit to the AC dimer has been postulated to require methylation of the $\mathrm{C}$ subunit for some of the B-subunit families [39]. However, conflicting results have been reported that may reflect differences in experimental design and will be discussed further. Studies wherein $\mathrm{PP} 2 \mathrm{~A} / \mathrm{B}$ is isolated from intact cells have revealed that methylation of the C subunit at L309 is required for incorporation of the $\mathrm{B}$ family of regulatory subunits into the holoenzyme complex [39, 40,42, 44, 54, 55]. Conversely, in vitro assembly of the PP2A/B holoenzyme complex does not require methylation of the $\mathrm{C}$ subunit for incorporation of the B family of regulatory subunits $[56,57]$. Similarly controversial, the requirement for C subunit L309 methylation was observed to be study-specific in in vitro $\mathrm{PP} 2 \mathrm{~A} / \mathrm{B}^{\prime}$ timer formation $[58,59]$. Methylation was dispensable for isolation of the PP2A/ $\mathrm{B}^{\prime}$ holoenzyme complex from intact cells [44]. The role of methylation of the $\mathrm{C}$ subunit in recruitment of $\mathrm{B}^{\prime \prime}$ and $\mathrm{B}^{\prime \prime \prime}$ families of regulatory subunits is less controversial with methylation of the $\mathrm{C}$ subunit being dispensable $[44,54]$. For more information, the reader is directed to an excellent recent paper [60].

Posttranslational modifications which influence formation of the PP2A holoenzyme complex also occur on the B subunit. $\mathrm{PP} 2 \mathrm{~A} / \mathrm{B}^{\prime}$ negatively regulates the ERK MAP kinase signal transduction pathway [61]. Through formation of a ternary complex of the early response gene product IEX-1, $B^{\prime} \gamma 1$, and ERK, ERK mediates its own disinhibition by phosphorylation of $B^{\prime} \gamma 1$ on $\mathrm{S} 327$ leading to $\mathrm{B}^{\prime} \gamma 1$ disassociation from the PP2A holoenzyme [62]. Since S327 is conserved among $\mathrm{B}^{\prime}$-subunit family members, it is likely that other $\mathrm{B}^{\prime}$ subunits are regulated similarly. Additionally, $\mathrm{B} \alpha$ is likely phosphorylated on S167 to disrupt the $B \alpha$ subunit from the AC dimer in early mitotic stages. However, PP2A/B $\alpha$ activity is necessary to resolve the mitotic spindles and conclude mitosis; therefore, autodephosphorylation may occur on $\mathrm{B} \alpha$ to allow efficient $\mathrm{PP} 2 \mathrm{~A} / \mathrm{B} \alpha$ heterotrimer formation and cell cycle progression [63]. Thus, phosphorylation of the Bregulatory subunits also influences holoenzyme assembly and, therefore, substrate specificity.

Phosphorylation of the B subunit of heterotrimeric PP2A also potentiates the catalytic activity of the holoenzyme complex. In response to activation of D1 dopamine receptors on striatal neurons, cAMP-dependent protein kinase A (PKA) phosphorylates $\mathrm{B}^{\prime} \delta$ at S566 increasing activity of the PP2A/ $\mathrm{B}^{\prime} \delta$ holoenzyme towards dopamine- and cAMPregulated neuronal phosphoprotein $(D A R P P-32)[64,65]$. Dephosphorylation of T75 on DARPP-32 by PP2A/B' $\delta$ disinhibits PKA-mediated phosphorylation of DARPP-32 at T34 which converts DARPP-32 into a potent PP1 inhibitor leading to changes in neuronal signaling. This circuit acts to attenuate phospho-T75 inhibition of T34 phosphorylation of $D A R P P-32$. This circuit has been shown to be differentially regulated by psychomotor stimulants and antipsychotics acting on different striatal neuron subpopulations [66]. 


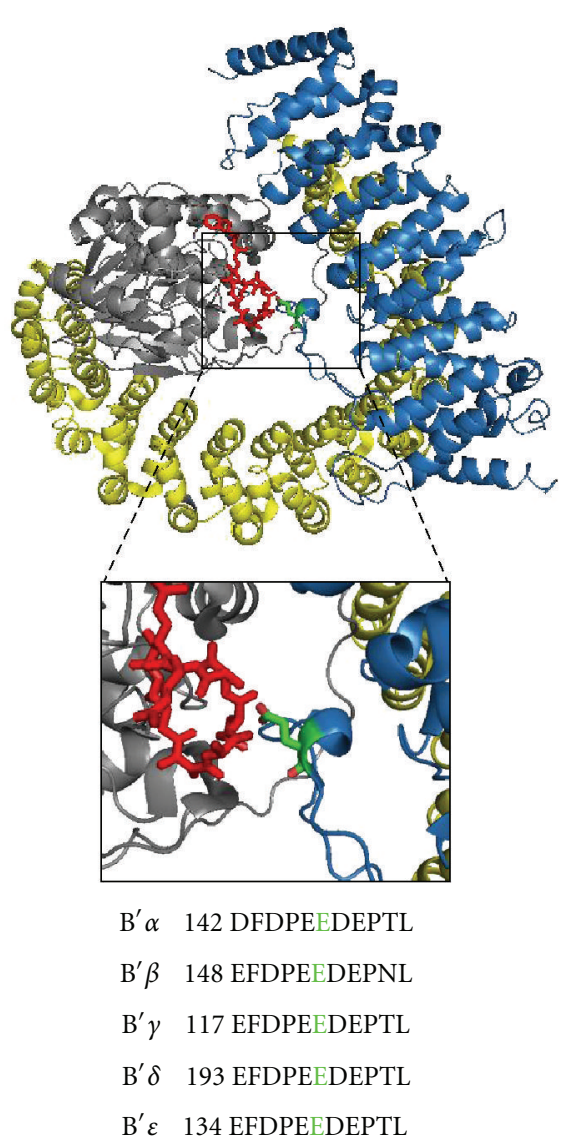

(a)

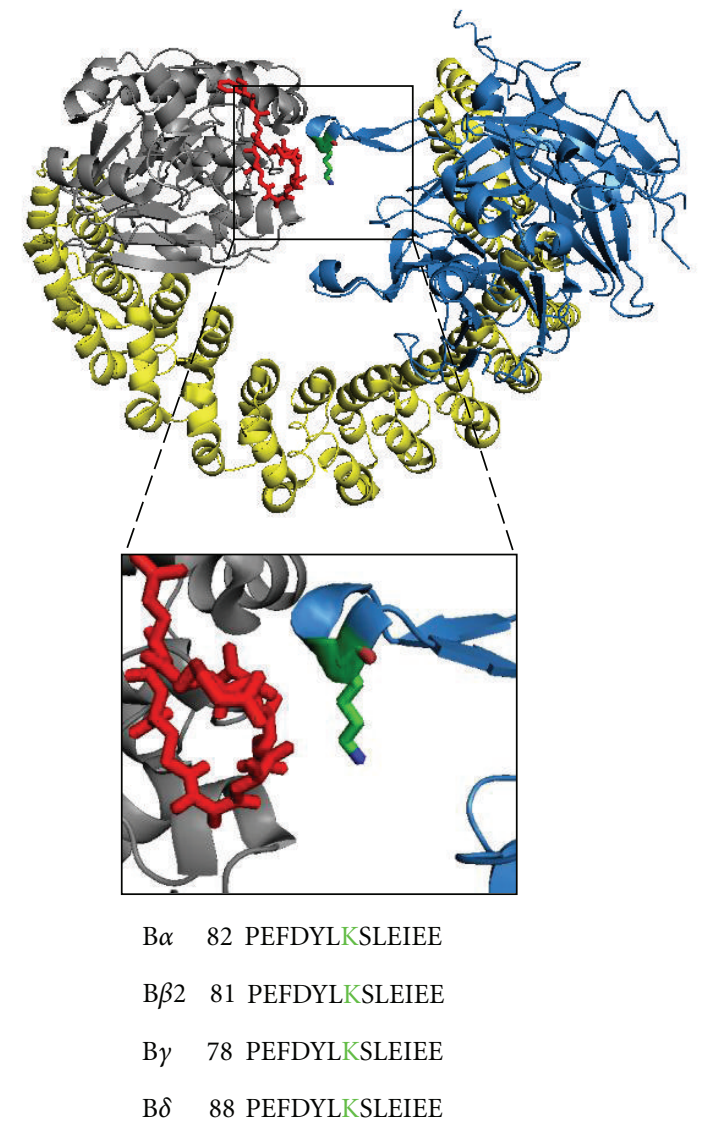

(b)

Figure 2: A portion of the $\mathrm{B}^{\prime}$-and B-regulatory subunits contacts the active site of the C-catalytic subunit. Ribbon diagrams showing a "top-down" view of two heterotrimeric holoenzyme PP2A complexes. The subunits of the holoenzyme complexes are color-coded with the catalytic C subunit in gray, the scaffold A subunit in yellow, and B subunits in blue. The PP2A inhibitor microcystin-LR is shown near the active site as a red stick figure. (a), Structure of the PP2A/B' $\gamma$ holoenzyme (PDB 2NPP); inset highlights the infiltration of the B' $\gamma$-subunit residue, E122 (green), into the catalytic core above. Below, sequence alignment of the $\mathrm{B}^{\prime}$ family of regulatory subunits with the conserved glutamate residue (green). (b), Structure of the PP2A/B $\alpha$ holoenzyme (PDB 3DW8); inset shows infiltration of the B $\alpha$-subunit residues, K88 (green), into the catalytic core above. Below, sequence alignment of the B family of regulatory subunits with the conserved lysine residue (green).

2.3. Regulating PP2A through Protein Inhibitors. While assembly of the many trimeric PP2A holoenzymes directs cellular localization and substrate specificity, further regulation is afforded through binding of specific protein inhibitors of PP2A. One such inhibitor SET $\left(\mathrm{I}_{2} / \mathrm{TAF}-1\right)$ is upregulated during the progression of chronic myelogenous leukemia through $\mathrm{BCR} / \mathrm{ABL}$ activity and results in decreased PP2A activity. Importantly, restoring PP2A activity prevents disease progression in an animal model of leukemogenesis [67]. An additional PP2A inhibitor is the protein CIP2A (cancerous inhibitor of PP2A), overexpression of which is associated with several human malignancies. CIP2A associates with c-myc to protect its phosphorylated S62 from PP2A-directed activity stabilizing the c-myc protein and allowing it to promote oncogenesis [68].

Recently an interplay between the Drosophila serine/ threonine kinase Greatwall (gwl) and $\mathrm{PP} 2 \mathrm{~A} / \mathrm{B} \delta$ was observed during mitotic entry in two separate studies $[69,70]$.
$\mathrm{PP} 2 \mathrm{~A} / \mathrm{B} \delta$ activity prevents mitotic entry by maintaining $\mathrm{Cdc} 25$ in a dephosphorylated and inactive state. Gwl reverses this inhibition through the phosphorylation of S67 of both $\alpha$-endosulfine (Ensa) and cyclic adenosine monophosphate(cAMP-) regulated phosphoprotein-19 (Arpp-19). Phosphorylation converts Ensa and Arpp-19 into very specific inhibitors of $\mathrm{PP} 2 \mathrm{~A} / \mathrm{B} \delta$ activity and produces activation of Cdc25 leading to cell cycle progression. Similar cell cycle regulatory activity has been observed with the mammalian ortholog of gwl, MASTL; however, the MASTL-PP2A interaction has yet to be characterized [71].

\subsection{Substrate Recruitment by Regulatory Subunit Interactions} Distant from the Active Site. Once targeted to specific subcellular locales, the PP2A holoenzyme must recruit and dephosphorylate target substrates. Recent structural studies have begun to suggest the mechanism by which the regulatory subunit of PP2A mediates initial binding to target substrates. 
The B family of regulatory subunits adopts a seven-bladed $\beta$-propeller structure [72]. Other $\beta$-propeller proteins have been shown to bind ligands in the central depression on the top surface of the toroid [73]. Crystallization of PP2A/B $\alpha$ revealed a cluster of acidic residues in this depression that is available to recruit potential substrates containing a basic motif [56]. In this same study, the acidic central depression of $\mathrm{B} \alpha$ was experimentally confirmed to bind the microtubuleassociated protein tau, an established $\mathrm{PP} 2 \mathrm{~A} / \mathrm{B} \alpha$ substrate. Several conserved aspartate and glutamate residues in $\mathrm{B} \alpha$ engage in weak, electrostatic interactions across a large basic portion of tau and support dephosphorylation of tau at multiple sites through cycles of binding and unbinding [56].

Structurally divergent $\mathrm{B}^{\prime}$ family members may recruit substrates in a similar fashion as the B family. The crystal structure of $\mathrm{PP} 2 \mathrm{~A} / \mathrm{B}^{\prime} \gamma$ shows that the $\mathrm{B}^{\prime}$ subunit contains 18 stacked $\alpha$-helices which adopt 8 huntingtin elongation A subunit Tor- (HEAT-) like repeats [58, 59]. A portion of these HEAT-like repeats interact with the A subunit of the holoenzyme to mediate regulatory subunit incorporation into the holoenzyme complex. Like the $\mathrm{B} \alpha$ subunit, an acidic patch is exposed in the $\mathrm{B}^{\prime}$ family of regulatory subunits and may mediate protein-protein interactions and substrate recruitment [59].

\subsection{Substrate Recruitment by Regulatory Subunit Interactions} Near the Active Site. Structural studies of PP2A have revealed a conserved loop in the $\mathrm{B}^{\prime}$ family of regulatory subunits which infiltrates the catalytic core of the holoenzyme $[58,59]$ (Figure 2(a)). At the tip of this loop is a conserved glutamate residue, E153 ( $\mathrm{B}^{\prime} \beta$ numbering), which contacts through its carbonyl oxygen the catalytic subunit and through its carboxyl group a cocrystallized microcystin molecule in the active site. Mutational analysis revealed that E153 is an absolute requirement for efficient dephosphorylation of tyrosine hydroxylase ( $\mathrm{TH}$ ), a known $\mathrm{PP} 2 \mathrm{~A} / \mathrm{B}^{\prime} \beta$ substrate, as well as other as yet unidentified cellular substrates of this PP2A holoenzyme [74]. Further, it was determined that E153 of $B^{\prime} \beta$ interacts with R37 and R38 of TH to mediate dephosphorylation of both S31 and S40 on TH. Positively charged residues in the vicinity of target phospho-serine/threonine residues could represent a consensus sequence for $\mathrm{B}^{\prime}$ subunit-mediated dephosphorylation. Further, the infiltrating loop is likely a conformationally dynamic structure which is not sterically hindered by surrounding portions of the A, C, or B subunits. Since R37/38 are important for dephosphorylation of both upstream (S40) and downstream (S31) sites, it appears that the orientation of phosphopeptides relative to the catalytic cleft is not constrained by additional interactions. Collectively, the above observations support a model in which the sites of interaction between the substrate and the $B$ regulatory subunit that are distant and near the active site together control substrate specificity. First, the interaction occurring at sites distant from the active site increases the local substrate concentration. Following this initial substrate recruitment, the interactions near the active site mediate site-specific dephosphorylation.

Although divergent in its sequence, an analogous structure from the $\beta$-propeller fold of the B family of regulatory subunits extends to the catalytic core of the holoenzyme [56] (Figure 2(b)). This loop places conserved residues of the B-family subunits very near the holoenzyme active site. Unpublished observations generated in our lab suggests that of these loop residues K87 of $\mathrm{B} \beta 2$ may play a similar role as E153 of $B^{\prime} \beta$ in site-specific dephosphorylation of target substrates; however, further characterization of this substrate specificity loop is required.

\section{Conclusion}

PP2A is a ubiquitous protein phosphatase responsible for the dephosphorylation of many different intracellular targets. The diverse repertoire of potential substrates for PP2A is imparted by the incorporation of one of fifteen unique B-regulatory subunits. Recent studies have increased our understanding of the mechanisms by which the B subunit imparts specificity to the holoenzyme complex. Through selective incorporation of the B-regulatory subunit, the holoenzyme complex is recruited to discrete subcellular locales which define the sphere of influence for the phosphatase. Secondly, interactions between endogenous inhibitors and specific PP2A heterotrimers further restrict phosphatase activity. As shown for the B family, regulatory subunits mediate low-affinity interactions with substrates to increase the local concentration of substrates. Through a flexible substrate selectivity loop which contacts the catalytic subunit, interactions between the regulatory subunit and phosphosubstrate may mediate multiple nearby dephosphorylation events. With the current structural information available for the PP2A complexes, future high-resolution studies will further define the molecular mechanism of PP2A substrate specificity. As general, inhibitors of PP2A are either clinically irrelevant or toxic, as in the case of the small molecule inhibitor microcystin, novel methods to increase the specificity of PP2A inhibition or activation must be developed. A clearer understanding of the PP2A substrate specificity mechanisms will serve as the foundation for rational drug design of selective inhibitors and activators of specific PP2A holoenzyme complexes.

\section{Acknowledgments}

This work was supported by National Institute of Health grants NS43254 (to Stefan Strack) and predoctoral Training Grant in Pharmacological Sciences T32GM067795 (to Andrew M. Slupe).

\section{References}

[1] T. Hunter, "Protein kinases and phosphatases: the yin and yang of protein phosphorylation and signaling," Cell, vol. 80, no. 2, pp. 225-236, 1995.

[2] J. V. Olsen, B. Blagoev, F. Gnad et al., "Global, in vivo, and sitespecific phosphorylation dynamics in signaling networks," Cell, vol. 127, no. 3, pp. 635-648, 2006.

[3] J. A. Sakoff and A. McCluskey, "Protein phosphatase inhibition: structure based design. Towards new therapeutic agents," Current Pharmaceutical Design, vol. 10, no. 10, pp. 1139-1159, 2004. 
[4] V. V. Vintonyak, A. P. Antonchick, D. Rauh, and H. Waldmann, "The therapeutic potential of phosphatase inhibitors," Current Opinion in Chemical Biology, vol. 13, no. 3, pp. 272-283, 2009.

[5] J. L. McConnell and B. E. Wadzinski, "Targeting protein serine/threonine phosphatases for drug development," Molecular Pharmacology, vol. 75, no. 6, pp. 1249-1261, 2009.

[6] J. S. Lazo and P. Wipf, "Phosphatases as targets for cancer treatment," Current Opinion in Investigational Drugs, vol. 10, no. 12, pp. 1297-1304, 2009.

[7] L. Martin, X. Latypova, and F. Terro, "Post-translational modifications of tau protein: implications for Alzheimer's disease," Neurochemistry International, vol. 58, no. 4, pp. 458471, 2011.

[8] E. Kickstein, S. Krauss, P. Thornhill et al., "Biguanide metformin acts on tau phosphorylation via mTOR/protein phosphatase 2A (PP2A) signaling," Proceedings of the National Academy of Sciences of the United States of America, vol. 107, no. 50, pp. 21830-21835, 2010.

[9] J. Lu, J. S. Kovach, F. Johnson et al., "Inhibition of serine/threonine phosphatase PP2A enhances cancer chemotherapy by blocking DNA damage induced defense mechanisms," Proceedings of the National Academy of Sciences of the United States of America, vol. 106, no. 28, pp. 11697-11702, 2009.

[10] F. Rusnak and P. Mertz, "Calcineurin: form and function," Physiological Reviews, vol. 80, no. 4, pp. 1483-1521, 2000.

[11] Y. Shi, "Serine/threonine phosphatases: mechanism through structure," Cell, vol. 139, no. 3, pp. 468-484, 2009.

[12] M. Bollen, W. Peti, M. J. Ragusa, and M. Beullens, "The extended PP1 toolkit: designed to create specificity," Trends in Biochemical Sciences, vol. 35, no. 8, pp. 450-458, 2010.

[13] J. Roy and M. S. Cyert, "Cracking the phosphatase code: docking interactions determine substrate specificity," Science Signaling, vol. 2, no. 100, p. re9, 2009.

[14] P. J. A. Eichhorn, M. P. Creyghton, and R. Bernards, "Protein phosphatase $2 \mathrm{~A}$ regulatory subunits and cancer," Biochimica et Biophysica Acta, vol. 1795, no. 1, pp. 1-15, 2009.

[15] V. Janssens and J. Goris, "Protein phosphatase 2A: a highly regulated family of serine/threonine phosphatases implicated in cell growth and signalling," Biochemical Journal, vol. 353, no. 3, pp. 417-439, 2001.

[16] R. Chenna, H. Sugawara, T. Koike et al., "Multiple sequence alignment with the Clustal series of programs," Nucleic Acids Research, vol. 31, no. 13, pp. 3497-3500, 2003.

[17] D. H. Huson, D. C. Richter, C. Rausch, T. Dezulian, M. Franz, and R. Rupp, "Dendroscope: an interactive viewer for large phylogenetic trees," BMC Bioinformatics, vol. 8, Article ID 460, 2007.

[18] D. M. Virshup and S. Shenolikar, "From promiscuity to precision: protein phosphatases get a makeover," Molecular Cell, vol. 33, no. 5, pp. 537-545, 2009.

[19] S. Strack, J. A. Zaucha, F. F. Ebner, R. J. Colbran, and B. E. Wadzinski, "Brain protein phosphatase $2 \mathrm{~A}$ : developmental regulation and distinct cellular and subcellular localization by B subunits," Journal of Comparative Neurology, vol. 392, no. 4, pp. 515-527, 1998.

[20] C. P. Flegg, M. Sharma, C. Medina-Palazon et al., "Nuclear export and centrosome targeting of the protein phosphatase $2 \mathrm{~A}$ subunit $\mathrm{B} 56 \alpha$ : role of $\mathrm{B} 56 \alpha$ in nuclear export of the catalytic subunit," Journal of Biological Chemistry, vol. 285, no. 24, pp. 18144-18154, 2010.

[21] B. McCright, A. M. Rivers, S. Audlin, and D. M. Virshup, "The B56 family of protein phosphatase 2A (PP2A) regulatory subunits encodes differentiation-induced phosphoproteins that target PP2A to both nucleus and cytoplasm," Journal of Biological Chemistry, vol. 271, no. 36, pp. 22081-22089, 1996.

[22] N. Bhasin, S. R. Cunha, M. Mudannayake, M. S. Gigena, T. B. Rogers, and P. J. Mohler, "Molecular basis for PP2A regulatory subunit B56 $\alpha$ targeting in cardiomyocytes," American Journal of Physiology, vol. 293, no. 1, pp. H109-H119, 2007.

[23] M. S. Gigena, A. Ito, H. Nojima, and T. B. Rogers, "A B56 regulatory subunit of protein phosphatase 2A localizes to nuclear speckles in cardiomyocytes," American Journal of Physiology, vol. 289, no. 1, pp. H285-H294, 2005.

[24] A. Ito, T. R. Kataoka, M. Watanabe et al., "A truncated isoform of the PP2A B56 subunit promotes cell motility through paxillin phosphorylation," EMBO Journal, vol. 19, no. 4, pp. 562-571, 2000.

[25] A. Ito, Y. I. Koma, M. Sohda et al., "Localization of the PP2A B56 $y$ regulatory subunit at the Golgi complex: possible role in vesicle transport and migration," American Journal of Pathology, vol. 162, no. 2, pp. 479-489, 2003.

[26] A. Ito, Y. I. Koma, K. Watabe et al., "A truncated isoform of the protein phosphatase $2 \mathrm{~A}$ B56 $\gamma$ regulatory subunit may promote genetic instability and cause tumor progression," American Journal of Pathology, vol. 162, no. 1, pp. 81-91, 2003.

[27] Y. I. Koma, A. Ito, K. Watabe, S. H. Kimura, and Y. Kitamura, "A truncated isoform of the PP2A B56y regulatory subunit reduces irradiation-induced $\mathrm{Mdm} 2$ phosphorylation and could contribute to metastatic melanoma cell radioresistance," Histology and Histopathology, vol. 19, no. 2, pp. 391-400, 2004.

[28] R. K. Dagda, J. A. Zaucha, B. E. Wadzinski, and S. Strack, "A developmentally regulated, neuron-specific splice variant of the variable subunit Bbeta targets protein phosphatase $2 \mathrm{~A}$ to mitochondria and modulates apoptosis," Journal of Biological Chemistry, vol. 278, no. 27, pp. 24976-24985, 2003.

[29] K. Schmidt, S. Kins, A. Schild, R. M. Nitsch, B. A. Hemmings, and J. Götz, "Diversity, developmental regulation and distribution of murine PR55/B subunits of protein phosphatase 2A," European Journal of Neuroscience, vol. 16, no. 11, pp. 2039-2048, 2002.

[30] R. K. Dagda, C. A. Barwacz, J. T. Cribbs, and S. Strack, "Unfolding-resistant translocase targeting: a novel mechanism for outer mitochondrial membrane localization exemplified by the $\mathrm{B} \beta 2$ regulatory subunit of protein phosphatase $2 \mathrm{~A}$," Journal of Biological Chemistry, vol. 280, no. 29, pp. 2737527382, 2005.

[31] R. K. Dagda, R. A. Merrill, J. T. Cribbs et al., "The spinocerebellar ataxia 12 gene product and protein phosphatase $2 \mathrm{~A}$ regulatory subunit $\mathrm{B} \beta 2$ antagonizes neuronal survival by promoting mitochondrial fission," Journal of Biological Chemistry, vol. 283, no. 52, pp. 36241-36248, 2008.

[32] Z. Jin, J. Shi, A. Saraf et al., "The $48-\mathrm{kDa}$ alternative translation isoform of PP2A:B56e is required for Wnt signaling during midbrain-hindbrain boundary formation," Journal of Biological Chemistry, vol. 284, no. 11, pp. 7190-7200, 2009.

[33] K. Zwaenepoel, J. Goris, C. Erneux, P. J. Parker, and V. Janssens, "Protein phosphatase 2A PR130/B" $\alpha 1$ subunit binds to the $\mathrm{SH} 2$ domain-containing inositol polyphosphate 5phosphatase 2 and prevents epidermal growth factor (EGF)induced EGF receptor degradation sustaining EGF-mediated signaling," FASEB Journal, vol. 24, no. 2, pp. 538-547, 2010.

[34] T. S. Kitajima, S. A. Kawashima, and Y. Watanabe, "The conserved kinetochore protein shugoshin protects centromeric cohesion during meiosis," Nature, vol. 427, no. 6974, pp. 510517, 2004. 
[35] A. W. Kerrebrock, D. P. Moore, J. S. Wu, and T. L. Orr-Weaver, "Mei-S332, a drosophila protein required for sister-chromatid cohesion, can localize to meiotic centromere regions," Cell, vol. 83, no. 2, pp. 247-256, 1995.

[36] T. S. Kitajima, T. Sakuno, K. I. Ishiguro et al., "Shugoshin collaborates with protein phosphatase 2 A to protect cohesin," Nature, vol. 441, no. 1, pp. 46-52, 2006.

[37] D. Clift, F. Bizzari, and A. L. Marston, "Shugoshin prevents eohesin cleavage by PP2A-dependent inhibition of separase," Genes and Development, vol. 23, no. 6, pp. 766-790, 2009.

[38] Z. Xu, B. Cetin, M. Anger et al., "Structure and function of the PP2A-shugoshin interaction," Molecular Cell, vol. 35, no. 4, pp. 426-441, 2009.

[39] J. C. Bryant, R. S. Westphal, and B. E. Wadzinski, "Methylated C-terminal leucine residue of PP2A catalytic subunit is important for binding of regulatory $\mathrm{B} \alpha$ subunit," Biochemical Journal, vol. 339, no. 2, pp. 241-246, 1999.

[40] H. Chung, A. C. Nairn, K. Murata, and D. L. Brautigan, "Mutation of Tyr307 and Leu309 in the protein phosphatase 2A catalytic subunit favors association with the $\alpha 4$ subunit which promotes dephosphorylation of elongation factor-2," Biochemistry, vol. 38, no. 32, pp. 10371-10376, 1999.

[41] E. Ogris, D. M. Gibson, and D. C. Pallas, "Protein phosphatase 2A subunit assembly: the catalytic subunit carboxy terminus is important for binding cellular B subunit but not polyomavirus middle tumor antigen," Oncogene, vol. 15, no. 8, pp. 911-917, 1997.

[42] T. Tolstykh, J. Lee, S. Vafai, and J. B. Stock, "Carboxyl methylation regulates phosphoprotein phosphatase $2 \mathrm{~A}$ by controlling the association of regulatory B subunits," EMBO Journal, vol. 19, no. 21, pp. 5682-5691, 2000.

[43] J. Wu, T. Tolstykh, J. Lee, K. Boyd, J. B. Stock, and J. R. Broach, "Carboxyl methylation of the phosphoprotein phosphatase 2A catalytic subunit promotes its functional association with regulatory subunits in vivo," EMBO Journal, vol. 19, no. 21, pp. 5672-5681, 2000.

[44] S. Longin, K. Zwaenepoel, J. V. Louis, S. Dilworth, J. Goris, and V. Janssens, "Selection of protein phosphatase $2 \mathrm{~A}$ regulatory subunits is mediated by the $\mathrm{C}$ terminus of the catalytic subunit," Journal of Biological Chemistry, vol. 282, no. 37, pp. 26971-26980, 2007.

[45] V. Nunbhakdi-Craig, S. Schuechner, J. M. Sontag et al., "Expression of protein phosphatase 2A mutants and silencing of the regulatory $\mathrm{B} \alpha$ subunit induce a selective loss of acetylated and detyrosinated microtubules," Journal of Neurochemistry, vol. 101, no. 4, pp. 959-971, 2007.

[46] J. Chen, B. L. Martin, and D. L. Brautigan, "Regulation of protein serine-threonine phosphatase type-2A by tyrosine phosphorylation," Science, vol. 257, no. 5074, pp. 1261-1264, 1992.

[47] I. De Baere, R. Derua, V. Janssens et al., "Purification of porcine brain protein phosphatase 2A leucine carboxyl methyltransferase and cloning of the human homologue," Biochemistry, vol. 38, no. 50, pp. 16539-16547, 1999.

[48] N. Leulliot, S. Quevillon-Cheruel, I. Sorel et al., "Structure of protein phosphatase methyltransferase 1 (PPM1), a leucine carboxyl methyltransferase involved in the regulation of protein phosphatase 2A activity," Journal of Biological Chemistry, vol. 279, no. 9, pp. 8351-8358, 2004.

[49] J. Lee, Y. Chen, T. Tolstykh, and J. Stock, "A specific protein carboxyl methylesterase that demethylates phosphoprotein phosphatase 2A in bovine brain," Proceedings of the National Academy of Sciences of the United States of America, vol. 93, no. 12, pp. 6043-6047, 1996.
[50] E. Ogris, X. Du, K. C. Nelson et al., "A protein phosphatase methylesterase (PME-1) is one of several novel proteins stably associating with two inactive mutants of protein phosphatase 2A," Journal of Biological Chemistry, vol. 274, no. 20, pp. 14382-14391, 1999.

[51] S. Longin, J. Jordens, E. Martens et al., "An inactive protein phosphatase $2 \mathrm{~A}$ population is associated with methylesterase and can be re-activated by the phosphotyrosyl phosphatase activator," Biochemical Journal, vol. 380, no. 1, pp. 111-119, 2004.

[52] S. Ortega-Gutiérrez, D. Leung, S. Ficarro, E. C. Peters, and B. F. Cravatt, "Targeted disruption of the PME-1 gene causes loss of demethylated PP2A and perinatal lethality in mice," PLoS ONE, vol. 3, no. 7, Article ID e2486, 2008.

[53] G. Kranias, L. F. Watt, H. Carpenter et al., "Protein phosphatase 2A carboxymethylation and regulatory B subunits differentially regulate mast cell degranulation," Cellular Signalling, vol. 22, no. 12, pp. 1882-1890, 2010.

[54] X. X. Yu, X. Du, C. S. Moreno et al., "Methylation of the protein phosphatase $2 \mathrm{~A}$ catalytic subunit is essential for association of $\mathrm{B} \alpha$ regulatory subunit but not SG2NA, striatin, or polyomavirus middle tumor antigen," Molecular Biology of the Cell, vol. 12, no. 1, pp. 185-199, 2001.

[55] J. Wu, T. Tolstykh, J. Lee, K. Boyd, J. B. Stock, and J. R. Broach, "Carboxyl methylation of the phosphoprotein phosphatase 2A catalytic subunit promotes its functional association with regulatory subunits in vivo," EMBO Journal, vol. 19, no. 21, pp. 5672-5681, 2000.

[56] Y. Xu, Y. Chen, P. Zhang, P. D. Jeffrey, and Y. Shi, "Structure of a protein phosphatase 2A holoenzyme: insights into B55mediated Tau dephosphorylation," Molecular Cell, vol. 31, no. 6, pp. 873-885, 2008.

[57] T. Ikehara, S. Ikehara, S. Imamura, F. Shinjo, and T. Yasumoto, "Methylation of the C-terminal leucine residue of the PP2A catalytic subunit is unnecessary for the catalytic activity and the binding of regulatory subunit (PR55/B)," Biochemical and Biophysical Research Communications, vol. 354, no. 4, pp. 1052-1057, 2007.

[58] U. S. Cho and W. Xu, "Crystal structure of a protein phosphatase 2A heterotrimeric holoenzyme," Nature, vol. 445, no. 7123, pp. 53-57, 2007.

[59] Y. Xu, Y. Xing, Y. Chen et al., "Structure of the protein phosphatase 2A holoenzyme," Cell, vol. 127, no. 6, pp. 12391251, 2006.

[60] V. Janssens, S. Longin, and J. Goris, "PP2A holoenzyme assembly: in cauda venenum (the sting is in the tail)," Trends in Biochemical Sciences, vol. 33, no. 3, pp. 113-121, 2008.

[61] W. Liu, A. M. Silverstein, H. Shu, B. Martinez, and M. C. Mumby, "A functional genomics analysis of the B56 isoforms of Drosophila protein phosphatase 2A," Molecular and Cellular Proteomics, vol. 6, no. 2, pp. 319-332, 2007.

[62] C. Letourneux, G. Rocher, and F. Porteu, "B56-containing PP2A dephosphorylate ERK and their activity is controlled by the early gene IEX-1 and ERK," EMBO Journal, vol. 25, no. 4, pp. 727-738, 2006.

[63] M. H. A. Schmitz, M. Held, V. Janssens et al., "Live-cell imaging RNAi screen identifies PP2A-B55 $\alpha$ and importin- $\beta$ 21 as key mitotic exit regulators in human cells," Nature Cell Biology, vol. 12, no. 9, pp. 886-893, 2010.

[64] J. H. Ahn, T. McAvoy, S. V. Rakhilin, A. Nishi, P. Greengard, and A. C. Nairn, "Protein kinase A activates protein phosphatase 2A by phosphorylation of the B56 $\delta$ subunit," Proceedings of the National Academy of Sciences of the United States of America, vol. 104, no. 8, pp. 2979-2984, 2007. 
[65] U. Y. Yu and J. H. Ahn, "Phosphorylation on the PPP2R5D B regulatory sub unit modulates the biochemical properties of protein phosphatase 2A," BMB Reports, vol. 43, no. 4, pp. 263 267, 2010.

[66] H. S. Bateup, P. Svenningsson, M. Kuroiwa et al., "Cell typespecific regulation of DARPP-32 phosphorylation by psychostimulant and antipsychotic drugs," Nature Neuroscience, vol. 11, no. 8, pp. 932-939, 2008.

[67] P. Neviani, R. Santhanam, R. Trotta et al., "The tumor suppressor PP2A is functionally inactivated in blast crisis CML through the inhibitory activity of the BCR/ABL-regulated SET protein," Cancer Cell, vol. 8, no. 5, pp. 355-368, 2005.

[68] M. R. Junttila, P. Puustinen, M. Niemelä et al., "CIP2A inhibits PP2A in human malignancies," Cell, vol. 130, no. 1, pp. 51-62, 2007.

[69] S. Mochida, S. L. Maslen, M. Skehel, and T. Hunt, "Greatwall phosphorylates an inhibitor of protein phosphatase $2 \mathrm{~A}$ that is essential for mitosis," Science, vol. 330, no. 6011, pp. 1670$1673,2010$.

[70] A. Gharbi-Ayachi, J.-C. Labbé, A. Burgess et al., "The substrate of Greatwall kinase, Arpp19, controls mitosis by inhibiting protein phosphatase 2A," Science, vol. 330, no. 6011, pp. 1673$1677,2010$.

[71] E. Voets and R. M. F. Wolthuis, "MASTL is the human orthologue of Greatwall kinase that facilitates mitotic entry, anaphase and cytokinesis," Cell Cycle, vol. 9, no. 17, pp. 35913601, 2010.

[72] S. Strack, R. Ruediger, G. Walter, R. K. Dagda, C. A. Barwacz, and J. Thomas Cribbs, "Protein phosphatase 2A holoenzyme assembly: identification of contacts between B-family regulatory and scaffolding a subunits," Journal of Biological Chemistry, vol. 277, no. 23, pp. 20750-20755, 2002.

[73] D. K. Wilson, D. Cerna, and E. Chew, “The 1.1-Å structure of the spindle checkpoint protein Bub3p reveals functional regions," Journal of Biological Chemistry, vol. 280, no. 14, pp. 13944-13951, 2005.

[74] A. Saraf, E. A. Oberg, and S. Strack, "Molecular determinants for PP2A substrate specificity: charged residues mediate dephosphorylation of tyrosine hydroxylase by the PP2A/B' regulatory subunit," Biochemistry, vol. 49, no. 5, pp. 986-995, 2010. 

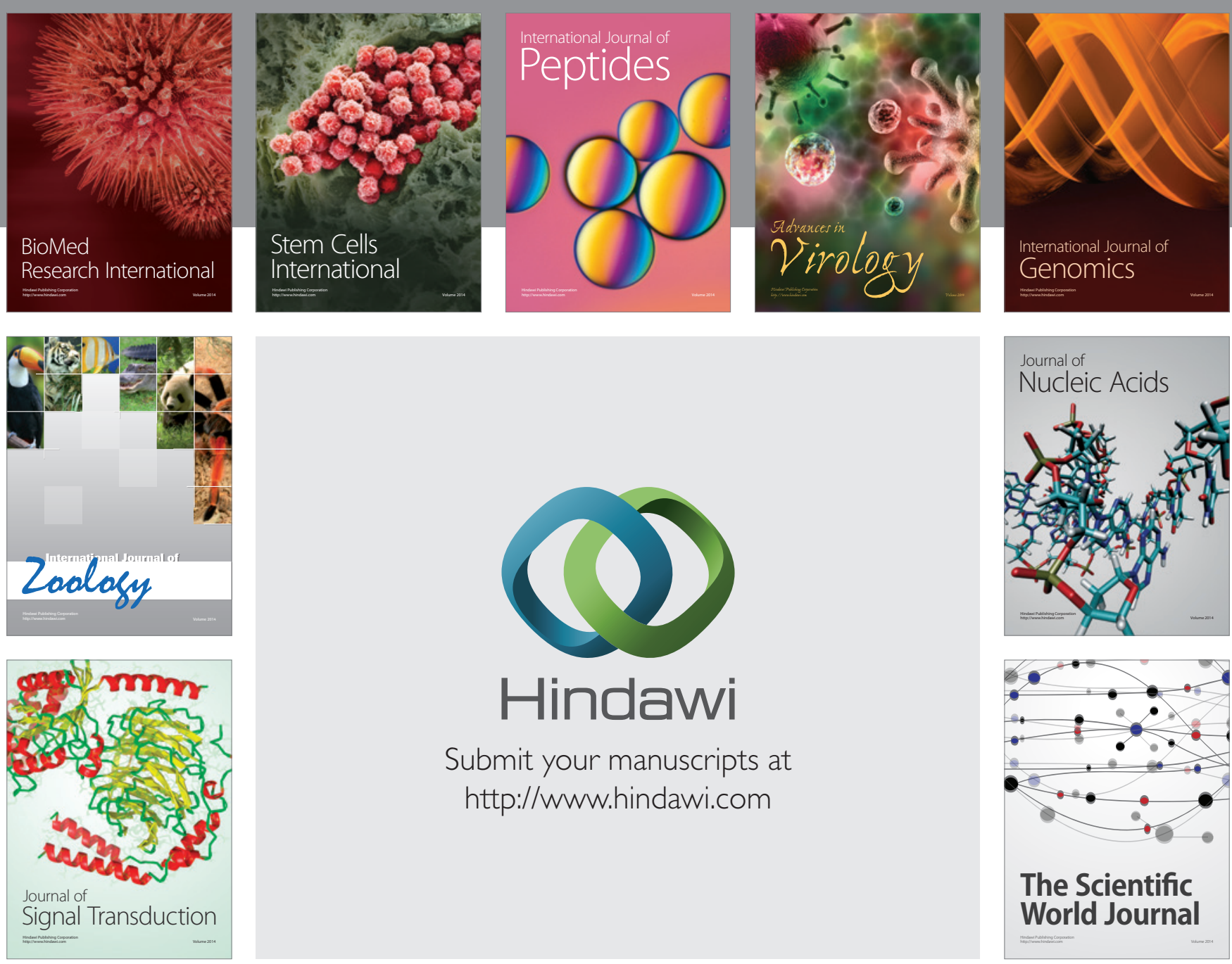

Submit your manuscripts at

http://www.hindawi.com
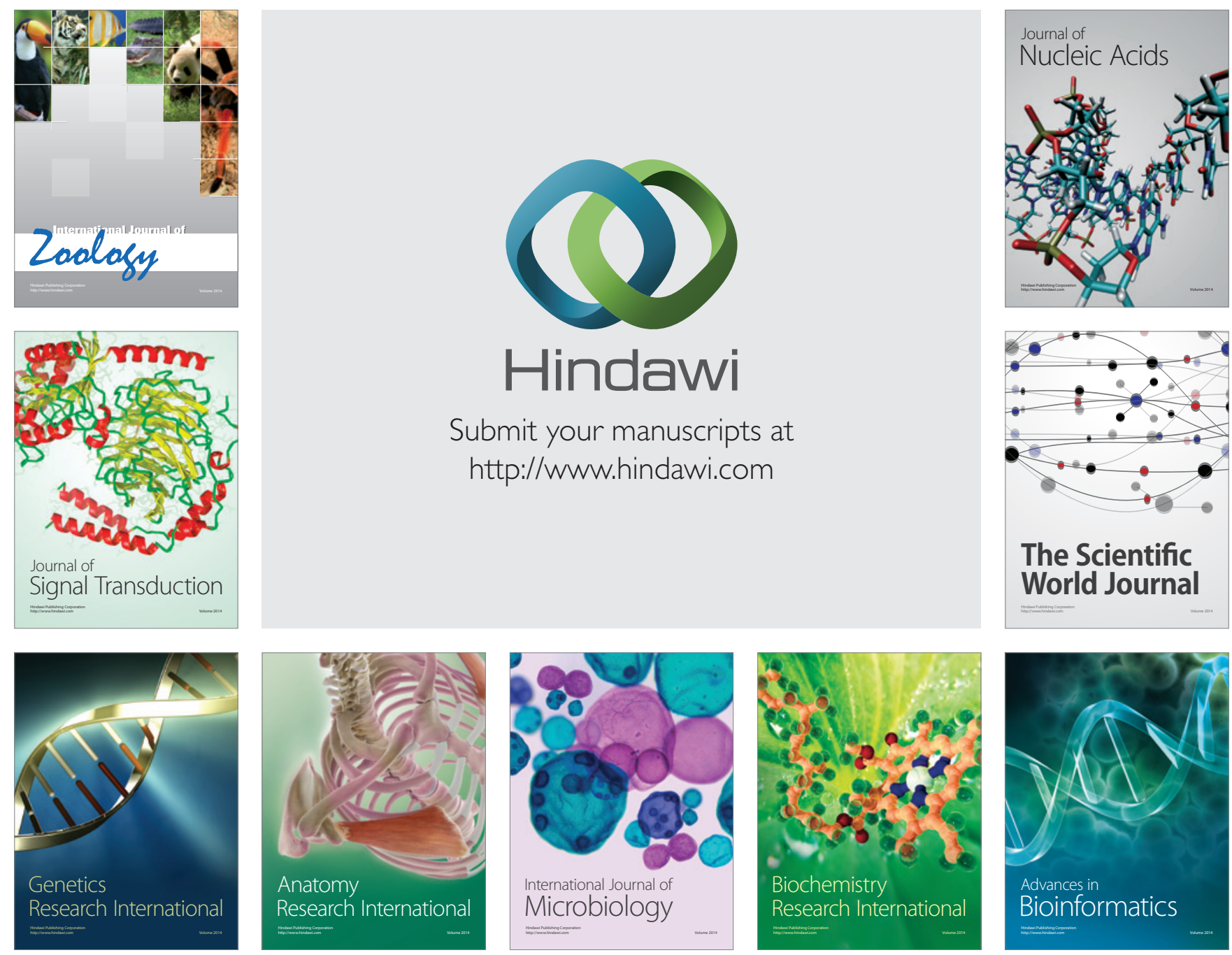

The Scientific World Journal
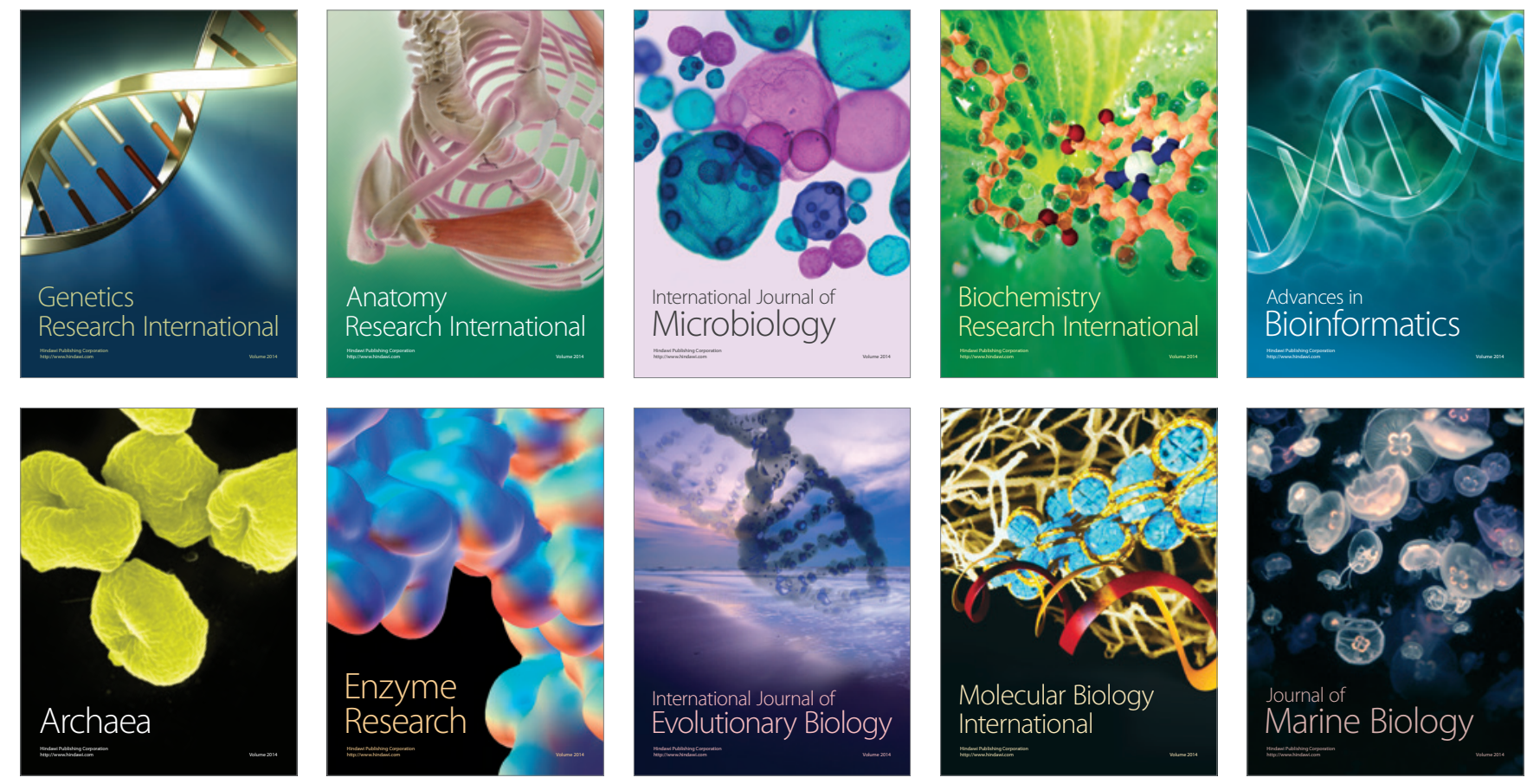